\title{
POLARIZED NEUTRON REFLECTION: A TECHNIQUE TO STUDY MAGNETIZATION PROFILES AT SURFACES AND INTERFACES
}

\author{
R. Felici \\ Istituto Struttura della Materia del C.N.R. \\ Via E. Fermi 38, 00044 Frascati, Rome, Italy
}

\begin{abstract}
The interaction of a neutron beam at grazing angle of incidence can be well described by the use of laws of classical optics when a refractive index is introduced. This follows from the undulatory nature of neutrons and from their interaction potential with matter. For polarized neutrons a magnetic material acts as a bircfringent material and a magnetization profile can be inferred from reflectivity measurements. In this paper we will briefly describe the theory of polarized neutron reflection and some applications of this technique to study superconductors, thin and granular magnetic films. PACS numbers: 75.25. $+\mathrm{z}$, 75.30.Pd, 75.60.Ej
\end{abstract}

\section{Introduction}

Thermal neutrons are widely used in condensed matler to study the properties of materials. Their associated wavelengths are comparable with the interatomic distances and their energies are similar to the collective atomic excitations. Their interaction potential with nuclei is very weak and can be approximated in a very simple manner making the analysis of the data simple. Neutrons are neutral particles but they have a spin, then they also interact by dipole interactions with magnetic atoms. Theory of neutron scattering and its application to the study of materials can be found elsewhere [1], but the purpose of this paper is to give some basis on a different technique: polarized neutron reflection. We will show that at grazing angle of incidence the interaction of a neutron beam with a surface can be described by the laws of classical optics after the introduction of an opportune refractive index and will give some examples on how polarized neutron reflection is able to determine magnetization profiles at surfaces and interfaces with a spatial resolution which goes from few angströms up to thousands of angströms. Examples will be reported in determining the magnetic penetration depth in superconductors, in studying the magnetization of very thin films and the difference in blocking temperature of the surface with respect to the bulk in the case of magnetic granular films. 


\section{Theory}

When applied to the propagation of the neutron radiation the terms reflection and refraction are not used only figuratively. These terms come from the strict analogy between the equations which describe the propagation of a neutron and its interaction with matter and those describing the propagation of an electromagnetic radiation.

Let us consider the diagram of Fig. 1 where a neutron beam impinges onto a surface with an angle of incidence $\vartheta_{i}$. In this simple case the interaction potential

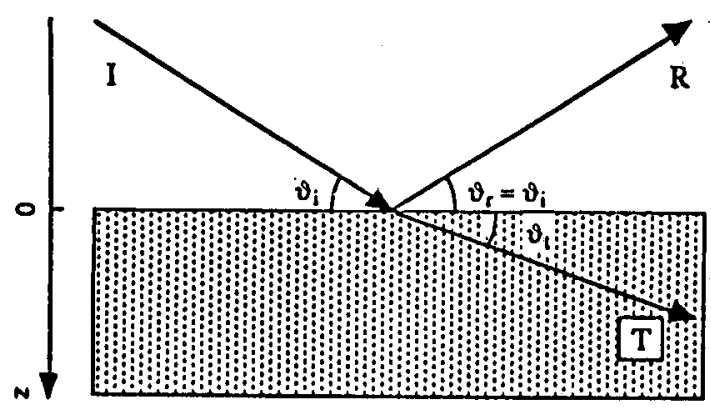

Fig. 1. Schematic diagram of the reflection of a neutron beam onto a surface with an angle of incidence $\vartheta_{i}$. In this case the refractive index of the material is less than one, which is true for most of the cases.

$V_{\text {opt }}$ is only a function of $z$, the distance from the surface. The propagation of the neutron [2] can be described by the Schrödinger equation for nonrelativistic particles which in the time independent form is

$$
\nabla^{2} \varphi+\frac{8 \pi^{2} m}{h^{2}}\left[E-V_{\mathrm{opt}}(z)\right] \varphi=0
$$

where $E$ and $m$ are the neutron energy and mass.

The propagation of an electromagnetic wave under similar conditions is described by the equation [3]:

$$
\nabla^{2} E+\left[n(z) k^{2}\right]^{2} E=0,
$$

where $n(z)$ is the refractive index of the sample and $k=\omega / c$ is module of the wave vector of the radiation. The mathematical analogy between Eqs. (1) and (2) is evident and as a consequence it results that the propagation of the neutron de Broglie wave in the potential $V_{\mathrm{opt}}(z)$ is analogous to the propagation of an electromagnetic radiation in a medium with a refractive index $n(z)$ [4]. By using the de Broglie relations for the energy and the wave vector module of a neutron: $E=h^{2} / 2 m \lambda^{2}$ and $k=\left(8 \pi 2 m E / h^{2}\right)^{1 / 2}$ and substituting these values in Eqs. (1) and (2) we define the refractive index of a material for neutrons as

$$
n(z)=\sqrt{1-\frac{V_{\mathrm{opt}}(z)}{E}} .
$$


The potential $V_{\text {opt }}$ used in Eq. (3) is not the interaction potential of a neutron with a single nucleus but it is obtained by an average of that potential along planes parallel to the surface. Neutrons do not have charge but they have a magnetic moment. Then their interaction potential with matter is the sum of two terms, one describing the nuclear interaction with nuclei and the other due to their magnetic interaction with the induction magnetic field inside the sample $\left(V(r)=V_{N}(r)+\right.$ $\left.V_{M}(r)\right)$. The nuclear term is well described by a series of the Fermi pseudopotential each centered in a nucleus position,

$$
V_{N}(r)=\sum_{i}\left(h^{2} / 2 \pi m\right) b_{i} \delta\left(r-r_{i}\right),
$$

where the $b_{i}$ are the scattering lengths and are characteristic quantities for each nucleus. In the case of a material constituted by different isotopes or nuclei we take the average scattering length because of the spatial average. The magnetic term is due to the interaction of the dipole moment of neutrons, $\mu_{n}$, with the induction magnetic field $\boldsymbol{B}$ inside the sample and it is given by

$$
V_{\mathrm{M}}(\boldsymbol{r})=-\mu_{\mathrm{n}} \cdot B(r) \text {. }
$$

In all the cases we will consider that the $B$ vector is always parallel to the surface, perpendicular to the exchanged momentum and the neutron magnetic moment is along the magnetic field direction. Moreover, the angle of reflection is so small that effects due to the magnetic form factor [1] are negligible. We can then write the following relation for the refractive index of polarized neutrons [4]:

$$
\begin{aligned}
n^{2}(z) & =1-\left[\frac{h^{2}}{2 \pi m} N(z)\langle b(z)\rangle \pm \mu_{\mathrm{n}} B(z)\right] E^{-1} \\
& =1-\frac{\lambda^{2}}{\pi}\left[N\langle b(z)\rangle \pm \frac{2 \pi m}{h^{2}} \mu_{\mathrm{n}} B(z)\right]
\end{aligned}
$$

where the signs \pm refer to the cases where neutron spins are parallel or antiparallel with respect to the magnetic field direction and $N(z)$ is the numerical density as a function of the distance from the surface. We have to notice that the refractive index of vacuum is not zero when there is a magnetic field. An immediate consequence of expression (4) is that magnetic materials are birefringent and that their optical properties strictly depend on the neutron spin state. This is also at the basis of the development of neutron polarizer mirrors, which offer the advantages of a wide polychromatic polarized neutron beam and a very high efficiency in terms of polarization as well as of transmission with respect to standard polarizers based on the diffraction from single crystals.

For almost all the materials the refractive index for neutrons is slightly smaller than unity (some parts in $10^{-6}$ ) and a material is optically less dense than vacuum. Then as a consequence of Snell's law, $\cos \vartheta_{\mathrm{i}} / \cos \vartheta_{\mathrm{t}}=n$, we have total reflection when the exchanged momentum is smaller than a critical value. This was first used by Fermi and co-workers to determine the scattering lengths of many nuclei [5]. In Table we report scattering lengths, numerical densities and critical angles calculated for a neutron wavelength of $1 \AA$ for some common materials. 


\section{TABLE}

Nuclear scattering lengths, numerical density and value of the critical angle for neutrons with a wavelength of $1 \AA$. In the case of titanium we never observe total reflection because of the negative scattering length value.

\begin{tabular}{c|l|l|c}
\hline \hline Material & $N\left[\AA^{-3}\right]$ & $b[\mathrm{fm}]$ & $\vartheta_{\mathrm{c}}$ [degrees] \\
\hline $\mathrm{Be}$ & 0.123 & 7.74 & 0.099 \\
$\mathrm{Al}$ & 0.0603 & 3.5 & 0.047 \\
$\mathrm{Ti}$ & 0.0566 & -3.4 & - \\
$\mathrm{Cu}$ & 0.0847 & 7.6 & 0.082 \\
${ }^{63} \mathrm{Cu}$ & 0.0847 & 6.7 & 0.077 \\
${ }^{65} \mathrm{Cu}$ & 0.0847 & 11.1 & 0.099 \\
$\mathrm{H}_{2} \mathrm{O}$ & 0.0335 & 1.68 & 0.024 \\
$\mathrm{SiO}_{2}$ & 0.0267 & 1.58 & 0.066
\end{tabular}

To calculate the magnetic part of the refractive index we can use two formulas depending on our own convenience. If the sample is made of magnetic atoms with a magnetic moment $\mu$, we can use

$$
\frac{2 \pi m}{h^{2}} \mu_{\mathrm{n}} B(z)=N c^{*}\langle\mu\rangle,
$$

where $\langle\mu\rangle$ is the component of the magnetic moment along the applied field direction and $c^{*}$ is a constant equal to $0.2645 \times 10^{-12} \mathrm{~cm} / \mu_{\mathrm{B}}$.

For a perfect diamagnet as a superconductor in the Meissner regime there is no magnetic field inside the sample apart from a small region close to the surface where the magnetic field strength decays almost exponentially with a characteristic length. In this case we can write [6]:

$$
\frac{2 \pi m}{h^{2}} \mu_{\mathrm{n}} B(z)=c B_{0}\left(1-\mathrm{e}^{-z / \lambda}\right),
$$

where $B_{0}$ is the applied magnetic field, $z$ is the distance from the surface, $A$ is the magnetic penetration depth and $c$ is a constant equal to $2.316 \times 10^{-10} \AA^{-2} /$ Oe.

Knowing the refractive index, we can use all the formalism developed for classical optics to calculate the reflectivity of a neutron beam as a function of the exchanged momentum $Q=(4 \pi / \lambda) \sin \vartheta$, where $\lambda$ is the neutron wavelength and $\vartheta$ is the reflection angle [7]. For instance, in the simple case of a sharp interface between two media as in Fig. 1, the reflectivity can be calculated by the simple Fresnel law

$$
R=\left|\frac{\sin \vartheta_{\mathrm{i}}-\sin \vartheta_{\mathrm{t}}}{\sin \vartheta_{\mathrm{i}}+\sin \vartheta_{\mathrm{t}}}\right|^{2}
$$

In Fig. 2 we report the calculated reflectivity from quartz together with the curves obtained by including an angular beam divergence and surface roughness 
corrections [8]. Real surfaces are in fact not sharp but they have dimensions of several angströms. This causes a decrease in the reflected beam intensity far from the critical edge.

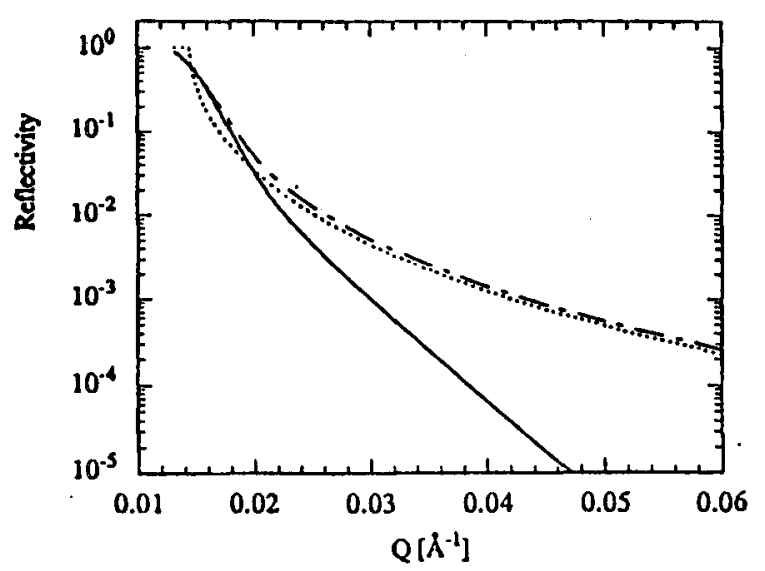

Fig. 2. Calculated neutron reflectivities for an air-quartz interface as a function of the exchanged momentum. The dotted line is the ideal profile, the dashed line is obtained by including an angular beam divergence of $15 \%$ and the solid line is after correction for a surface roughness of $50 \AA$.

When the system is not so simple but the refractive index is a more complicated expression of $z$, the reflectivity can always be calculated either using the matrix method [9] or solving the following set of equations [10]:

$$
R(Q)=\left|\frac{r_{1}+r(0)}{r_{1}+r_{1} r(0)}\right|^{2}
$$

with

$$
\begin{aligned}
& r_{1}=\frac{p(0)-p_{\mathrm{v}}}{p(0)+p_{\mathrm{v}}} \\
& p(z)=\sqrt{n^{2}(z)-\cos ^{2} \vartheta_{\mathrm{i}}},
\end{aligned}
$$

where the quantities $p(z)$ are the sine of the angle of the neutron beam with respect to the surface as a function of the distance from the surface, $p(0)$ is for $z=0$ and $p_{\mathrm{v}}$ is $\sin \vartheta_{\mathrm{i}}$. All the dependence of the reflectivity on the refractive index is contained in the term $r(0)$ which is defined by the following integral equation:

$$
r(0)=-\int_{0}^{\infty} \frac{p^{\prime}(z)}{2 p(z)}\left\{\left[1-r^{2}(z)\right] \exp \left[-\frac{4 \pi \mathrm{i}}{\lambda} \int_{0}^{z} p(t) \mathrm{d} t\right]\right\} \mathrm{d} z
$$

where $p^{\prime}(z)$ is the derivative of $p(z)$ with respect to $z$.

The analytical solution of this equations is not always feasible and in general we prefer to calculate the reflectivity using the matrix method. Anyway this expression allows to understand how the spin dependent reflectivities are influenced 
by the magnetization profile. In fact, expanding Eq. (8) for a homogeneous material $(b(z)=$ const $)$, far from the critical edge $\left(Q \gg Q_{c}\right.$ or $\lambda \ll \lambda_{\mathrm{c}}$ or $\left.\vartheta \gg \vartheta_{\mathrm{c}}\right)$ and when the magnetic component of the refractive index is much smaller than the nuclear part, we obtain for the spin dependent reflectivities the approximate form

$$
R^{ \pm}=R_{\mathrm{N}}\left|1 \pm c \int_{0}^{\infty} B^{\prime}(z) \exp \left(\frac{4 \pi \mathrm{i}}{\lambda} z p_{\mathrm{N}}\right) \mathrm{d} z\right|^{2},
$$

wherc $B^{\prime}(z)$ is the derivative of the modulus of the magnetic induction field with respect to the distance from the surface $z$ and the index $N$ refers to all the same quantities calculated using only the nuclear part of the refractive index. The difference between the spin dependent neutron reflectivities strictly depends on the Fourier transform of the derivative of the induction magnetic ficld with respect to the distance from the surface.

\section{Expcrimental apparatus}

The application of neutron reflection to the study of surfaces and interfaces is rapidly growing and at the moment almost every neutron source has or plans to have a neutron reflectometer in general dedicated to study surfaces of liquids. This implies having a titled bearn with respect to the horizontal. On the other hand, there are very few instruments fully dedicated to perform polarized neutron reflectivity measurements and, as an example, we will describe in this paragraph the polarized version of the CRISP reflectometer [11] installed at the ISIS neutron spallation source of the Rutherford Appleton Laboratory, see Fig. 3.

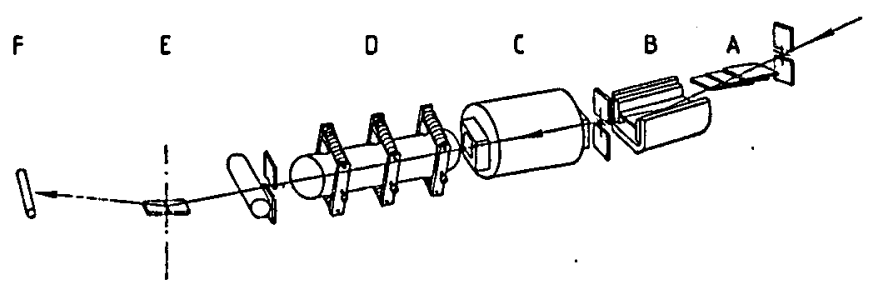

Fig. 3. Diagram of the CRISP reflectometer. (A) Frame overlap mirrors to avoid neutrons with wavelength longer than $13 \AA$; (B) neutron polarizer made of Co-Ti neutron supermirror; (C) non-adiabatic spin flipper; (D) magnetic guide; (E) sample position; (F) ${ }^{3} \mathrm{He}$ detector.

The reflection of neutrons is an elastic process in which the neutron does not change its energy. At a pulsed neutron source, like ISIS, it is possible to design a fixed geometry neutron reflectometer using the white incident beam and discriminating the neutron wavelength by the time-of-flight technique. We remind that the velocity of a thermal neutron is $v[\mathrm{~km} / \mathrm{s}]=3.955 / \lambda[\AA]$. This fact simplifies enormously the data collection with respect to steady neutron source where a monochromatic incident beam is used and the reflectivities are measured as a function of the reflection angle. In this latter case, in fact, a lot of care must be 
used to avoid that the sample would not intercept all the incident beam making absolute measurements impossible.

In this instrument the incident white spectrum is polarized by a Co-Ti supermirror. The useful wavelengths range is $2 \div 6.5 \AA$. The lower limit is defined by the critical edge of the polarizer, while the upper one by the source-detector distance $(12 \mathrm{~m})$ and the source repetition frequency $(50 \mathrm{~Hz})$. In order to measure both the spin dependent reflectivities a non-adiabatic spin flipper is used to reverse the neutron spin direction with respect to the magnetic field. In the whole used range both the polarization and fipper efficiency are better than $98 \%$ [12]. In order to a void spurious counts coming from very long wa velength neutrons generated by previous pulses it is necessary to put in the beam a mirror which together with a $50 \mathrm{~Hz}$ chopper take out from the beam all the neutrons with a wavelength longer than $6.5 \AA$.

The sample is situated on a goniometer between the poles of an electromagnet, then the applied magnetic field can be continuously varied from about $0.0005 \mathrm{~T}$ to about $0.350 \mathrm{~T}$. The alignment is performed by using a laser beam and the reproducibility of the reflection angle is better than $0.002^{\circ}$. A special cryostat has been designed in order to perform measurements down to $5 \mathrm{~K}$ having optical windows for sample alignment.

There are two types of detectors: a ${ }^{3}$ IIe proportional counter or a position sensitive detector. In general, the ${ }^{3} \mathrm{He}$ detector has a lower background and its use is preferred when it is not necessary to measure out of reflection intensity. The detector can be moved in order to set the reflectivity angle from a minimum value of $0.25^{\circ}$ up to few degrees.

\section{Results}

Polarized neutron reflection has been usefully employed to study surfaces magnetization profiles in ferromagnets, superconductors, superparamagnetic materials and magnetic superlattices. In this paragraph we will outline some experimental results. In general, the data are presented as the ratio of the spin dependent reflectivities which, as shown in Eq. (10), strictly depends on the Fourier transform of the derivative of the magnetization profile with respect to the distance from the surface.

Measurements made on ferromagnetic films of iron oxide [13] $\left(\mathrm{Fe}_{3} \mathrm{O}_{4}\right.$ and $\gamma-\mathrm{Fe}_{2} \mathrm{O}_{3}$ ) which are of important technological interest because they can be employed as recording media show some of the properties of the polarized neutron reflection technique. In general, the magnetic properties of $\gamma-\mathrm{Fe}_{2} \mathrm{O}_{3}$ are better than $\mathrm{Fe}_{3} \mathrm{O}_{4}$, because it has a higher coercive field and a higher residual magnetization. On the other hand, the films of $\gamma-\mathrm{Fe}_{2} \mathrm{O}_{3}$ obtained by oxidizing a $\mathrm{Fe}_{3} \mathrm{O}_{4}$ film at high temperature show very poor recording quality. Conventional magnetization measurements cannot explain this difference. Measurements of polarized neutron reflection (see Fig. 4) have shown the presence of a magnetic dead layer $150 \AA$ thick which exists at the surface of the $\gamma-\mathrm{Fe}_{2} \mathrm{O}_{3}$.

A superconductor for applied field lower than a critical value shows a perfect diamagnetism, the Meissner effect. In this case the magnetic field is restricted 


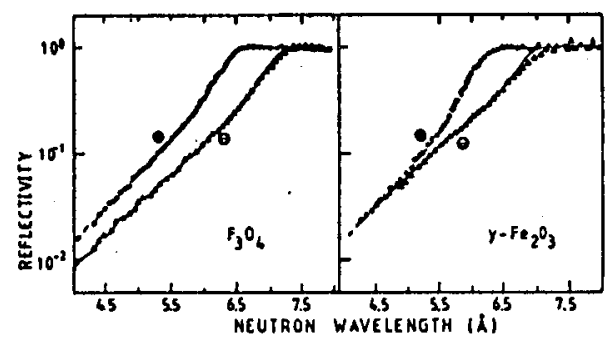

Fig. 4. Reflectivities curves for neutron polarized parallel ( + , circles) or antiparallel (-, squares) with respect to the applied field for (a) $\mathrm{Fe}_{3} \mathrm{O}_{4}$ and (b) $\gamma-\mathrm{Fe}_{2} \mathrm{O}_{3}$. The lines are the calculated reflectivity assuming a magnetic dead layer of $150 \AA$ for the $\gamma-\mathrm{Fe}_{2} \mathrm{O}_{3}$.

only to a region close to the surface and its strength decays almost exponentially with respect to the distance from the surface with a characteristic length $\Lambda$ the magnetic penetration deptl. This quantity is strictly related to the density of superconductors electrons [14] and its direct measurement is of fundamental importance. Polarized neutron reflcction is one of the few techniques able to determine it in a direct manner. By standard magnetization methods it is only possible to measure $\mathrm{d} \Lambda / \mathrm{d} T$ but there is no way to determine the constant of integration $\Lambda(0)$ and an absolute measurement of $A$ is then impossible. In Fig. 4 we report the spin reflectivity ratio for a niobium film in the Meissner regime [5]. By the fit of the data a value of $430 \AA$ at $4.6 \mathrm{~K}$ was obtained, which extrapolated to $0 \mathrm{~K}$ brings to the value for $A(0)$ of $410 \pm 10 \AA$ or $397 \pm 10 \AA$ depending on the theory. These values are in very good agreement with the values which were calculated by band theory. Measurements have been performed also on high- $T_{\mathrm{c}}$ superconductors, but these measurements do not agree with each other. The obtained value for $\Lambda$ is in the range between few hundred $\AA$ up to two thousand $\AA[15,16]$.

This technique has also been used in the study of the magnetic properties of very thin magnetic films. Experiments have been performed on sample constituted by $\mathrm{Fe}$ or Co few monolayers thick on $\mathrm{Cu}$ [17]. The samples were always covered with a layer of about $100 \AA$ of $\mathrm{Cu}$. This overlayer is a protection for the thin layer and it is essential for the neutron measurements. In fact, to amplify the magnetic signal the Fresnel interference of this overlayer is used. The amplitude of the fringes strongly depends on the magnetic part of the refractive index of the thin films and in this way it is possible to determine its magnetization. We have to remind that this technique is sensitive only to the magnetization along the surface and not to the perpendicular one.

Recently polarized neutron reflection is also been used in the study of magnetic superlattices. With this technique it is possible to determine simultaneously the thickness, the magnetization and the average interface dimension of each layer. In general, it is not obvious that the dimensions of the chemical superlattice are the same as of the magnetic. Moreover, with this technique it is also possible to discriminate between antiferromagnetism inside or among the magnetic layers.

Granular magnetic films are composite materials made of small magnetic 
particles dispersed in an amorphous alumina matrix. They are important because of possible technological applications and from a fundamental point of view for their similarity to spin glasses. The magnetic properties of these materials have been carefully studied by AC and DC susceptibility measurements and by the Mössbauer spectroscopy. The data can be interpreted by introducing a privileged

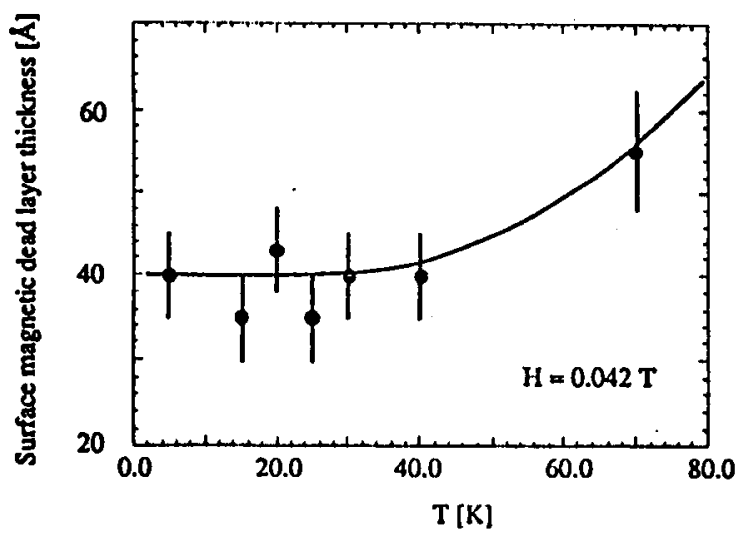

Fig. 5. Surface magnetic dead layer thickncss of a $\mathrm{Fe}-\mathrm{Al}_{2} \mathrm{O}_{3}$ granular film as a function of temperature. The blocking temperature for this material is at about $25 \mathrm{~K}$. The results indicate that the surface has a blocking temperature lower than the bulk one. The line is a guide for the eye.

direction for the grain magnetic moment and an anisotropy energy. Measurements of polarized neutron reflection were performed on two samples made of iron grains dispersed in an amorphous alumina matrix. The two samples had a different iron concentration and different average grain dimension.

From the data we were able to determine absolute magnetization curves with respect to temperature and applied ficld, in good agreement with theories and with previous standard magnetization results. Moreover, we were able to show that the thickness of the magnetic dead layer existing at the surface increases for temperature higher than the blocking one, as displayed in Fig. 5.

\section{Conclusions}

In this paper we have tried to outline the theory of polarized neutron reflection. We have shown that the ratio between the two neutron spin dependent reflectivities is related by an optical transform to the derivative of the induction magnetic field with respect to the surface. This technique which has been only in the recent years fully developed has many field of application either for a technological utilization and for fundamental physics studies. In the near future, it will probably become one of the most powerful tools that scientists have to study magnetization at surfaces. 


\section{References}

[1] G.E. Bacon, Neutron Diffraction, Clarendon Press, Oxford 1975.

[2] L.D. Landau, E.M. Lifshitz, Quantum Mechanics, 3rd ed., Pergamon Press, Oxford 1981.

[3] J.D. Jackson, Classical Electrodynamics, 2nd ed., Wiley, New York 1975.

[4] A.G. Klein, S.A. Werner, Rep. Prog. Phys. 46, 259 (1983).

[5] E. Fermi, L. Marshall, Phys. Rev. 71, 666 (1947).

[6] G.P. Felcher, R.T. Kampwirth, K.E. Gray, R. Felici, Plıys. Rev. Lett. 52, 1539 (1984).

[7] J. Lekner, Theory of Reflection, Marlinus Nijholf, Dordrecht 1987.

[8] L. Nèvot, P. Croce, Rev. Phys. Appl. 15, 761 (1980).

[9] M. Born, E. Wolf, Principles of Optics, Pergamou Press, Oxford 1970.

[10] G.P. Felcher, R. Felici, R.T. Kampwirth, K.E. Gray, J. Appl. Phys., 57, 3789 (1985).

[11] R. Felici, J. Penfold, R.C. Ward, W.G. Williams, Appl. Phys. A 45, 169 (1988).

[12] R. Felici, J. Penfold, R.C. Ward, W.G. Williams, Nucl. Instrum. Melhods Phys. Res. A 260, 309 (1987).

[13] S.S. Parkin, R. Sigsbee, R. Felici, G.P. Felcher, Appl. Phys. Lett. 48, 604 (1986).

[14] M. Tinkham, Introduction to Superconductivily, McGraw-IIill, New York 1975.

[15] R. Felici, J. Penfold, R.C. Ward, E. Olzi, F.C. Matacotta, Nature 329, 523 (1987).

[16] G.P. Felcher, private communication.

[17] J.A.C. Bland, D. Pescia, R.F. Willis, Phys. Rev. Lclt. 58 , 1244 (1987). 